\title{
Shoshin beriberi: a rare diagnostic problem
}

Sir,

The description by Engbers and colleagues of a case of profound circulatory collapse in an alcoholic (1984; 51: 581-2) is interesting as a description of recovery from severe overindulgence in alcohol, but the contention that the entire clinical presentation was due to thiamine deficiency is not supported by the biochemical data presented. The low erythrocyte transketolase activity should be ascribed to thiamine deficiency only if it is corrected by the addition of thiamine pyrophosphate in vitro. When this test was carried out the increase in transketolase activity was within the normal range. If the addition of thiamine pyrophosphate does not significantly increase the transketolase activity, it is difficult to sustain the argument that the patient is thiamine deficient.

As the authors state, depressed transketolase activity may occur in alcoholics with liver disease, and in patients with longstanding thiamine deficiency, but it has not been shown that the synthesis of transketolase is regulated by the availability of thiamine. Low transketolase activity in the presence of excess thiamine pyrophosphate may well be caused by abnormalities due to chronic alcoholism which are independent of thiamine deficiency. Our understanding of the relation between thiamine deficiency and heart failure will be improved only when more direct assays (for example, the thiochrome method ${ }^{1}$ ) are in routine use.

Mark Dancy,

M K Gaitonde,

J D Maxwell,

St George's Hospital,

London SW17 0QT.

\section{Reference}

1 Dancy M, Evans G, Gaitonde, MK, Maxwell JD. Blood thiamine and thiamine phosphate ester concentrations in alcoholic and non-alcoholic liver diseases. $\mathrm{Br} M e d ~ \mathcal{F} 1984$; 289: 79-82.

This letter was shown to Dr Engbers and colleagues, who reply as follows:

Sir,

We agree with Dr Dancy and colleagues that thiamine deficiency in our patient is not proved biochemically by a high thiamine pyrophosphate effect. As they state, the low transketolase activity in chronic alcoholism may be independent of thiamine deficiency. The thiamine pyrophosphate effect is clearly not a sensitive test to diagnose thiamine deficiency, which calls for a more direct assay.

In spite, however, of the lack of direct biochemical evidence of thiamine deficiency it can safely be concluded from the clinical picture of our patient that overindulgence in alcohol cannot be its only cause. This is because of the high cardiac output with a predominantly right sided heart failure and the rapid recovery after treatment with thiamine alone, while he had already ceased to take any alcohol two weeks before admission.
J G Engbers,
G P Molhoek,
A C Arntzenius
Department of Cardiology,
Leiden University Hospital,
Leiden,
The Netherlands.

\section{Prevalence of coronary artery disease in patients with isolated aortic valve stenosis}

Sir,

In their recent paper $(1984 ; 51: 121)$ Exadactylos et al refer to my paper on "Angina, coronary disease, and aortic stenosis" as having been published only in abstract form but never later in full. In fact, the study was published in full in the American Heart fournal (1977; 93: 382). This paper has been cited repeatedly by authors on this subject, including Thompson et al in the British Heart fournal (1979; 42: 447).

With reference to one of the issues discussed, our experience since 1977 has confirmed that significant coronary artery disease can be present in patients with aortic stenosis without angina.

E William Hancock,

Cardiology Division,

Stanford University Medical Center,

Stanford,

California 94305,

USA. 
Sir,

Exadactylos et al (1984; 51: 121-4) found three vessel disease only in patients with typical angina. In this group, its prevalence (on their sensitive criterion of $50 \%$ luminal narrowing in two projections) was $7 \%$. In the light of present knowledge, concurrent aortocoronary bypass grafting (in the hope of increasing survival) can be justified only in these patients and any others with left main stenosis. Aortic valve replacement alone almost always suffices for the relief of angina. The additional operative myocardial ischaemic time needed for concurrent coronary grafting is not without its hazards. ${ }^{1}$

A similarly low prevalence of prognostically important coronary disease may account for the very good survival of 52 consecutive patients treated for calcific aortic stenosis by aortic valve replacement alone at this hospital between 1973 and 1975. There was no hospital mortality in this group, only five of whom had preoperative coronary arteriography. Coronary perfusion was not used intraoperatively. After $7 \cdot 3$ years their cumulative survival was $90 \%$, three of the five deaths being due to non-cardiac causes. While 32 of these patients had angina at presentation, only one reported (non-disabling) angina during the postoperative follow up period.

If the low prevalence of important coronary disease reported by Exadactylos et al is general, it might be very difficult to show that routine coronary arteriography and bypass grafting are truly advantageous to the management of patients with typical aortic stenosis, many of whom require aortic valve replacement without avoidable delay.

W R Fitzgerald,

S M Bailey,

D Wainwright Evans.

Regional Cardiac Unit, Papworth Hospital, Cambridge CB3 9RE.

\section{Reference}

1 Ormerod OJM, McGregor CGA, Stone DL, Wisbey C,
Petch MC. Arrhythmias after coronary bypass surgery. $\mathrm{Br}$ Heart F 1984; 51: 618-21.

These letters were shown to the senior author of this paper who replies:

Sir,

I can only apologise most humbly for our failure to quote your full paper rather than the abstract from it. I note that you have now found significant coronary artery disease in patients with aortic stenosis without angina. This, of course, was inevitable. We are all familiar with painless ischaemia, and there is no reason at all why it should not occur also in patients with aortic stenosis without coronary artery disease. Nevertheless, wherever doing the best for the most still has to count the message is valid. Not many patients who have both severe aortic stenosis and significant coronary artery disease are going to be angina free, and a great many coronary arteriograms will have to be done in order to enable those few patients to have coronary bypass grafts.

In reply to the letter from Dr Fitzgerald and his colleagues, who point out that aortic valve replacement alone almost always results in relief of angina, I agree with this but do not share his experience of a low prevalence of later coronary deaths in patients who had undergone aortic valve replacement for aortic stenosis without preoperation coronary angiography or coronary bypass grafting for significant coronary disease. Others have been persuasive in their defence of coronary grafting at the time of aortic valve replacement not only to prevent subsequent coronary events but also to reduce operative mortality.

I believe that our middle of the road stand with coronary angiography in aortic stenosis only for patients in whom coronary artery disease is suspected is a reasonable one.

Celia M Oakley,

Cardiothoracic Institute,

Royal Postgraduate Medical School,

London W12 0HS.

\section{Notices}

\section{Cardiac pacing and electrophysiology}

The Third Asian Pacific Symposium on Cardiac Pacing and Electrophysiology will be held from 27 to 30 October 1985 at the Melbourne Hilton, Melbourne. Further information may be obtained from: Secretary-General, 3rd Asian-Pacific Symposium on Cardiac Pacing and Electrophysiology, Department of Cardiology, Suite 3.5, Epworth Medical Centre, 62 Erin Street, Richmond 3121, Victoria, Australia.

\section{British Cardiac Society}

The Autumn Meeting will be held at the Wembley Conference Centre, London, on 3 and 4 December 1984 , and the closing date for receipt of abstracts was 15 August 1984.

The Annual General Meeting for 1985 will take place in Birmingham on 17 and 18 April 1985, and the closing date for receipt of abstracts will be 8 January 1985. 